\title{
Asymmetric Zn-Aldol Reaction of Methyl Vinyl Ketone and Its Synthetic Applications
}

\author{
Barry M. Trost*, Seunghoon Shin, Joseph A. Sclafani \\ Department of Chemistry, Stanford University, Stanford, California 94305-5080
}

\section{Supporting Information}

General All reactions were performed under nitrogen atmosphere unless otherwise indicated. Acetone was distilled from Drierite ${ }^{\circledR}$. Ether and methylene chloride $\left(\mathrm{CH}_{2} \mathrm{Cl}_{2}\right)$ were purified on an alumina column solvent purification system, and tetrahydrofuran (THF) was distilled from sodium benzophenone ketyl. Methyl vinyl ketone was purchased from Aldrich and was distilled over anhydrous calcium chloride prior to use. Diethylzinc was purchased from Aldrich (Sure/Seal bottle, either 1.0 M in hexane or 1.1 $\mathrm{M}$ in toluene). Standard phenyl ligand (1) was synthesized as reported before. ${ }^{3 \mathrm{e}}$

Flash chromatography was performed with EM Science silica gel (0.040-0.063 mm grade) or 200 mesh Florisil (Aldrich). Analytical thin layer chromatography was performed with $0.2 \mathrm{~mm}$ coated commercial silica gel plates (E. Merck, DC Plastifolien, kieselgel 60 F254). Proton and broad-band decoupled ${ }^{13} \mathrm{C}$ nuclear magnetic resonance data were acquired on a Varian GEM-300, Mercury-400 or Unity Inova-500 spectrometer as indicated. Chemical shifts are reported in ppm relative to TMS as an internal standard. Infrared (IR) data were recorded in sodium chloride plates on PerkinElmer Paragon 500 FTIR spectrometer. Elemental analyses were performed by M-H-W Laboratories, Phoenix, Arizona. High resolution mass spectra (HRMS) were obtained from the Mass Spectrometry Resource, School of Pharmacy, University of California-San Francisco on a Kratos MS9 spectrometer. All compounds were judged to be pure $(>95 \%)$ on the basis of $1 \mathrm{H}$ NMR except where explicitly indicated otherwise. Proton and carbon NMR spectra are provided in the second Supporting Information document for all new compounds that do not have elemental analysis given.

Scheme A. Generation of Dinuclear Zn Catalyst
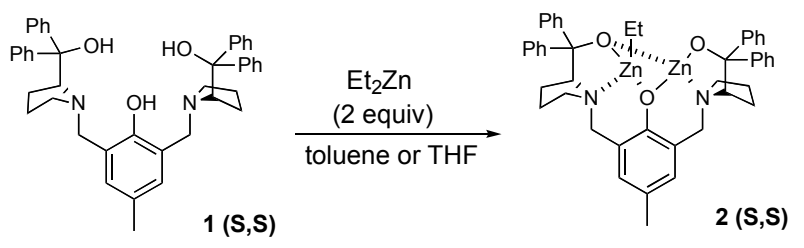

\section{Conditions for Table 1}

Typical condition (entry 3, Method A, $[$ ald $]=0.3 \mathrm{M},[\mathrm{MVK}]=7 \mathrm{M}$ )

Under nitrogen atmosphere, benzene $(\sim 0.5 \mathrm{~mL})$ was syringed into a test tube capped with rubber septa containing standard phenyl ligand $1(32 \mathrm{mg}, 0.05 \mathrm{mmol})$. After the tube was immersed in an ice-bath, the tube was carefully connected to a high vacuum $(\sim 1$ torr $)$ via needle. Then the bath was removed and evaporation was continued until solvent was removed completely. This drying process was repeated once more. To the resulting white solid was added THF $(0.5 \mathrm{~mL})$ and then a solution of diethyl zinc $(100 \mu \mathrm{L}$ of $1.0 \mathrm{M}$ soln in hexane, $0.10 \mathrm{mmol})$ was added dropwise over $5 \mathrm{~min}$ at ambient temperature. After being stirred at the same temperature for 20-30 min, a solution of catalyst was obtained $(\sim 0.1 \mathrm{M}$ in THF).

Reaction: Under nitrogen atmosphere, the solution of catalyst prepared as mentioned above was added via syringe to a mixture of freshly distilled (over anhydrous calcium chloride) methyl vinyl ketone $(1.0 \mathrm{~mL}, 12 \mathrm{mmol})$, isopropanol $(0.20 \mathrm{~mL}, 2.5 \mathrm{mmol}), 3$-benzyloxy-2,2-dimethylpropionaldehyde $(96.1$ $\mathrm{mg}, 0.5 \mathrm{mmol})$, and $4 \mathrm{~A}$ molecular sieve $(100 \mathrm{mg})$ at $-78{ }^{\circ} \mathrm{C}$. After the addition, the reaction mixture was 
immersed in the cold bath for the amount of time designated. After the reaction, the reaction was quenched with $0.1 \mathrm{~N} \mathrm{HCl}(2 \mathrm{~mL})$, and the aqueous phase was extracted with ether $(2 \mathrm{~mL} \times 4)$. The combined organic layers were dried over sodium sulfate. The solvent was removed under reduced pressure using rotary evaporator with ice-bath. The residue was loaded on a silica gel column (pretreatred with $\left.\mathrm{Et}_{3} \mathrm{~N}\right)$ and was eluted with $\mathrm{EtOAc} / \mathrm{pet}=1 / 8 \sim 1 / 5$ to give $83.7 \mathrm{mg}(64 \% \mathrm{y})$ of aldol, $25.6 \mathrm{mg}$ $(27 \%)$ of recovered starting material, and $25.6 \mathrm{mg}(21 \%)$ of elimination product.

Typical Condition (entry 9, Method B, [ald] = 0.8 M, $[\mathrm{MVK}]=5 \mathrm{M}$ )

Catalyst Preparation:Under nitrogen atmosphere, benzene $(\sim 0.5 \mathrm{~mL})$ was syringed into a test tube capped with rubber septa containing standard phenyl ligand (32 $\mathrm{mg}, 0.05 \mathrm{mmol})$. After the tube was immersed in an ice-bath, the tube was carefully connected to a high vacuum ( $\sim 1$ torr $)$ via needle. Then the bath was removed and evaporation was continued until solvent was removed completely. This drying process was repeated once more. To the resulting white solid was added anhydrous toluene $(0.25 \mathrm{~mL})$, then a solution of diethyl zinc $(91 \mu \mathrm{L} \text { of } 1.1 \mathrm{M} \text { soln in toluene, } 0.1 \mathrm{mmol})^{1}$ was added dropwise over 5 min at ambient temperature. After being stirred at the same temperature for 20-30 min, a solution of catalyst was obtained (ca. $0.2 \mathrm{M}$ in toluene).

Reaction: Under nitrogen atmosphere, aldehyde $(101.2 \mathrm{mg}, 0.5 \mathrm{mmol})$ was syringed into a tube with rubber septa containing freshly distilled (over anhydrous calcium chloride) methyl vinyl ketone $(0.25 \mathrm{~mL}, 3.0 \mathrm{mmol})$, isopropanol $(0.2 \mathrm{~mL}, 2.5$ equiv), and $4 \mathrm{~A}$ molecular sieve (100 $\mathrm{mg})$ at room temperature. To the resulting mixture cooled at $-78{ }^{\circ} \mathrm{C}$, catalyst solution prepared above was added dropwise over $2 \mathrm{~min}$. The tube was sealed with parafilm and was immersed in the cold bath for the amount of time designated $\left(-15^{\circ} \mathrm{C}, 14 \mathrm{~h}\right)$. A similar workup procedure and chromatography afforded the aldol product (67.8 mg, $50 \%$ ), starting aldehyde (32.5 mg, $32 \%)$, and elimination product (12\%).

Table A. Chiral HPLC Separation Conditions for the Aldol Adducts

\begin{tabular}{|c|c|c|}
\hline \multirow{2}{*}{ Compound $^{\mathrm{a}}$} & \multicolumn{2}{|c|}{ Column (Hept/IPA) } \\
\hline & Major isomer $(\mathrm{min})$ & Minor isomer (min) \\
\hline & \multicolumn{2}{|c|}{ OD $(98 / 2), 1 \mathrm{~mL} / \mathrm{min}$} \\
\hline & 10.2 & 8.5 \\
\hline & \multicolumn{2}{|c|}{ OD $(99 / 1), 1 \mathrm{~mL} / \mathrm{min}$} \\
\hline & 23.6 & 17.8 \\
\hline & \multicolumn{2}{|c|}{ OD $(99.5 / 0.5), 1 \mathrm{~mL} / \mathrm{min}$} \\
\hline & 18.0 & 16.2 \\
\hline & \multicolumn{2}{|c|}{ OJ $(97 / 7), 1 \mathrm{~mL} / \mathrm{min}$} \\
\hline & 14.7 & 17.9 \\
\hline & \multicolumn{2}{|c|}{$\mathrm{AD}(99.5 / 0.5), 0.9 \mathrm{~mL} / \mathrm{min}$} \\
\hline & 15.3 & 18.1 \\
\hline & \multicolumn{2}{|c|}{ OJ $(99.5 / 0.5), 1 \mathrm{~mL} / \mathrm{min}$} \\
\hline & 8.82 & 10.19 \\
\hline & \multicolumn{2}{|c|}{ OJ $(98 / 2), 1 \mathrm{~mL} / \mathrm{min}$} \\
\hline & 20.6 & 22.4 \\
\hline & \multicolumn{2}{|c|}{ OD $(99 / 1), 1 \mathrm{~mL} / \mathrm{min}$} \\
\hline & 9.69 & 7.27 \\
\hline & \multicolumn{2}{|c|}{ OD $(99.5 / 0.5), 1 \mathrm{~mL} / \mathrm{min}$} \\
\hline & 24.3 & 20.7 \\
\hline
\end{tabular}

${ }^{a}$ Absolute stereochemistry was determined by Mandelate method (Trost, B.M. et al. J.Org.Chem. 1986, $51,2370)$ 


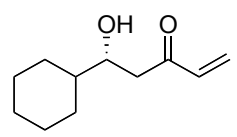

Entry 1, Table 1

${ }^{1} \mathrm{H}$ NMR $\left(400 \mathrm{~Hz}, \mathrm{CDCl}_{3}\right): \delta 6.38(\mathrm{dd}, J=10.5,17.4 \mathrm{~Hz}, \mathrm{H}), 6.25(\mathrm{dd}, J=1.3,17.3 \mathrm{~Hz}, \mathrm{H}), 5.89(\mathrm{dd}, J=$ 1.1, $11.3 \mathrm{~Hz}, \mathrm{H}$ ), 3.88 (br s, H), 2.97 (d, J = 1.8 Hz, H), 2.78 (dd, J=7.8, $17.0 \mathrm{~Hz}, \mathrm{H}$ ), 2.61 (dd, $J=3.8$, $16.4 \mathrm{~Hz}, \mathrm{H}), 1.92-0.80(\mathrm{~s}, 11 \mathrm{H}) .{ }^{13} \mathrm{C} \mathrm{NMR}\left(125 \mathrm{~Hz}, \mathrm{C}_{6} \mathrm{D}_{6}\right): \delta 200.8,136.8,128.2,71.5,43.4,43.3,29.2$, 28.1, 26.8, 26.5, 26.4, Anal. Calcd for $\mathrm{C}_{11} \mathrm{H}_{18} \mathrm{O}_{2}$ : C, 72.49; H, 9.95, found C, 72.27; H, 9.47.

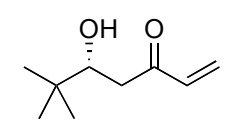

\section{Entry 2, Table 1}

$[\alpha]_{\mathrm{D}}^{22}=+41$ (c $0.89 \mathrm{CHCl}_{3}$ ). IR (neat): 3501, 2958, 2908, 2872, 1681, 1616, 1480, 1402, 1365, 1295, 1245, 1192, 1171, 1075, 1010, 986, $916 \mathrm{~cm}^{-1} .{ }^{1} \mathrm{H} \mathrm{NMR}\left(300 \mathrm{~Hz}, \mathrm{CDCl}_{3}\right): \delta 6.38(\mathrm{dd}, J=10.3,17.5 \mathrm{~Hz}$, H), $6.25(\mathrm{dd}, J=1.5,17.5 \mathrm{~Hz}, \mathrm{H}), 5.88(\mathrm{dd}, J=1.2,10.3 \mathrm{~Hz}, \mathrm{H}), 3.77$ (ddd, $J=2.0,3.2,10.0 \mathrm{~Hz}, \mathrm{H}), 2.93$ $(\mathrm{d}, J=3.4 \mathrm{~Hz}, \mathrm{H}), 2.81(\mathrm{dd}, J=2.0,17.0 \mathrm{~Hz}, \mathrm{H}), 2.61(\mathrm{dd}, J=10.0,17.1 \mathrm{~Hz}, \mathrm{H}), 0.83(\mathrm{~s}, 9 \mathrm{H}) .{ }^{13} \mathrm{C} \mathrm{NMR}$ $\left(75 \mathrm{MHz}, \mathrm{CDCl}_{3}\right): \delta 201.9,136.8,128.9,74.8,41.0,34.3,25.7$. Chiral HPLC assay: $\mathrm{t}_{\mathrm{r}}=15.2$ (major), 18.3 (minor) $\min$ (Chiralcel AD, $\lambda=230 \mathrm{~nm}$, heptane : isopropanol $=99 / 1,1 \mathrm{~mL} / \mathrm{min}$ ). HRMS calcd for $\mathrm{C}_{9} \mathrm{H}_{17} \mathrm{O}_{2}[\mathrm{M}+\mathrm{H}]:$ : 157.1229 , found 157.1227 .<smiles>C=CC(=O)CC(O)C(C)(C)COCc1ccccc1</smiles>

\section{Entry 3, Table 1}

$[\alpha]_{\mathrm{D}}^{23}=+31.4\left(\mathrm{c} 1.87 \mathrm{CHCl}_{3}\right)$. IR (neat): 3499, 3089. 3063, 3030, 2963, 2874, 1681, 1615, 1496, 1477, $1454,1402,1363,1308,1206,1096,1027,988,917,738,698 \mathrm{~cm}^{-1} .{ }^{1} \mathrm{H} \mathrm{NMR}\left(500 \mathrm{~Hz}, \mathrm{CDCl}_{3}\right): \delta 7.37-$ $7.25(\mathrm{~m}, 5 \mathrm{H}), 6.38(\mathrm{dd}, J=10.7,17.6 \mathrm{~Hz}, \mathrm{H}), 6.22(\mathrm{dd}, J=1.0,17.6 \mathrm{~Hz}, \mathrm{H}), 5.84(\mathrm{dd}, J=1.0,10.7 \mathrm{~Hz}$, $\mathrm{H}), 4.50\left(\mathrm{ABq}, v_{\mathrm{A}}=1.0, v_{\mathrm{B}}=9.3 \mathrm{~Hz}, 2 \mathrm{H}\right), 4.07(\mathrm{td}, J=3.2,9.3 \mathrm{~Hz}, \mathrm{H}), 3.42(\mathrm{~d}, J=3.7 \mathrm{~Hz}, \mathrm{H}), 3.35$ $\left(\mathrm{ABq}, v_{\mathrm{A}}=9.0, v_{\mathrm{B}}=19.3 \mathrm{~Hz}, 2 \mathrm{H}\right), 2.71(\mathrm{dd}, J=7.3,16.8 \mathrm{~Hz}, \mathrm{H}), 2.67(\mathrm{dd}, J=3.0,16.5 \mathrm{~Hz}, \mathrm{H}), 0.97(\mathrm{~s}$, $3 \mathrm{H}), 0.92(\mathrm{~s}, 3 \mathrm{H}) .{ }^{13} \mathrm{C} \mathrm{NMR}\left(125 \mathrm{MHz}, \mathrm{CDCl}_{3}\right): \delta 201.1,138.1,136.9,128.6,128.4,127.6,127.5,78.4$, 73.6, 73.5, 41.6, 38.2, 22.1, 19.9. Chiral HPLC assay: $\mathrm{t}_{\mathrm{r}}=13.8$ (major), 17.8 (minor) min (Chiralcel OJ, $\lambda$ $=254 \mathrm{~nm}$, heptane $:$ isopropanol $=97 / 3,1.0 \mathrm{~mL} / \mathrm{min})$. LRMS calcd for $\mathrm{C}_{16} \mathrm{H}_{23} \mathrm{O}_{3}: 263.1647$, found 263.2.<smiles>C=CC(=O)C[C@@H](O)C(C)(C)CO[Mg]C(C)(C)C</smiles>

\section{Entry 4, Table 1}

$[\alpha]_{\mathrm{D}}{ }^{26}=+31.1\left(\mathrm{c} 0.5 \mathrm{CHCl}_{3}\right) \cdot \mathrm{t}_{\mathrm{r}}=15.28$ (major), 18.08 (minor) min (Chiralcel AD, $\lambda=230 \mathrm{~nm}$, heptane : isopropanol $=99.5 / 0.5,1.0 \mathrm{~mL} / \mathrm{min}$ ). IR (neat): 3500, 2957, 2858, 1682, 1616, 1472, 1403, 1362, 1255, 1095, 990, 837, 764, $668 \mathrm{~cm}^{-1} .{ }^{1} \mathrm{H}$ NMR $\left(400 \mathrm{~Hz}, \mathrm{CDCl}_{3}\right): \delta 6.41(\mathrm{dd}, J=10.5,17.7 \mathrm{~Hz}, \mathrm{H}), 6.25(\mathrm{dd}, J=$ 1.2, $17.7 \mathrm{~Hz}, \mathrm{H}), 5.84(\mathrm{dd}, J=1.2,10.2 \mathrm{~Hz}, \mathrm{H}), 4.07$ (td, $J=3.0,9.6 \mathrm{~Hz}, \mathrm{H}), 3.65(\mathrm{~d}, J=3.0 \mathrm{~Hz}, \mathrm{H}), 3.48$ $(\mathrm{d}, J=1.2 \mathrm{~Hz}, 2 \mathrm{H}), 2.76(\mathrm{dd}, J=9.3,15.6 \mathrm{~Hz}, \mathrm{H}), 2.66(\mathrm{dd}, J=2.5,15.6 \mathrm{~Hz}, \mathrm{H}), 0.92(\mathrm{~s}, 3 \mathrm{H}), 0.89$ (s, 9H), $0.84(\mathrm{~s}, 3 \mathrm{H}), 0.06(\mathrm{~s}, 6 \mathrm{H}) .{ }^{13} \mathrm{C} \mathrm{NMR}\left(75 \mathrm{MHz}, \mathrm{CDCl}_{3}\right): \delta 201.0,136.9,128.5,74.2,71.9,41.9,38.4$, 25.8, 21.7, 19.2, 18.1, -5.7. Anal calcd for $\mathrm{C}_{15} \mathrm{H}_{30} \mathrm{O}_{3} \mathrm{Si}$, C: 62.89 , H: 10.55, found C: 62.93, H: 11.39 .

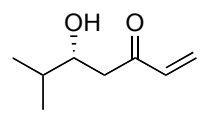

\section{Entry 5, Table 1}

$[\alpha]_{\mathrm{D}}^{25}=+35.7\left(\mathrm{c} 1.72 \mathrm{CHCl}_{3}\right)$. IR (neat): 3474, 2963, 1682, 1615, 1470, 1403, 1190, 1085, 1048, $992 \mathrm{~cm}^{-}$ 1. ${ }^{1} \mathrm{H}$ NMR $\left(500 \mathrm{~Hz}, \mathrm{CDCl}_{3}\right): \delta 6.35(\mathrm{dd}, J=10.4,17.7 \mathrm{~Hz}, \mathrm{H}), 6.25(\mathrm{dd}, J=1.0,17.6 \mathrm{~Hz}, \mathrm{H}), 5.88(\mathrm{dd}, J$ $=1.0,10.4 \mathrm{~Hz}, \mathrm{H}), 3.85(\mathrm{td}, J=2.7(\mathrm{~d}), 6.2(\mathrm{t}) \mathrm{Hz}, \mathrm{H}), 3.03(\mathrm{~d}, J=3.2 \mathrm{~Hz}, \mathrm{H}), 2.77(\mathrm{~d}, J=2.4,17.4 \mathrm{~Hz}$, H), $2.66(\mathrm{dd}, J=9.5,17.4 \mathrm{~Hz}, \mathrm{H}), 1.76-1.66(\mathrm{~m}, \mathrm{H}), 0.95(\mathrm{~d}, J=6.7 \mathrm{~Hz}, 3 \mathrm{H}), 0.92(\mathrm{~d}, J=6.7 \mathrm{~Hz}, 3 \mathrm{H})$. ${ }^{13} \mathrm{C}$ NMR $\left(125 \mathrm{MHz}, \mathrm{CDCl}_{3}\right): \delta 201.8,136.7,129.1,72.1,42.7,33.0,18.4,17.8$. Chiral HPLC assay: $\mathrm{t}_{\mathrm{r}}=$ 17.8 (minor), 23.6 (major) $\min$ (Chiralcel OD, $\lambda=230 \mathrm{~nm}$, heptane : isopropanol =99/1, $1.0 \mathrm{~mL} / \mathrm{min}$ ). HRMS calcd for $\mathrm{C}_{8} \mathrm{H}_{15} \mathrm{O}_{2}[\mathrm{M}+\mathrm{H}]$ : 143.1082, found 143.1072. 


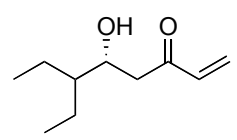

\section{Entry 6, Table 1}

$[\alpha]_{\mathrm{D}}^{25}=+11.1\left(\mathrm{c} 1.80 \mathrm{CHCl}_{3}\right)$. IR (neat): 3486, 2963, 2934, 2876, 1681, 1615, 1463, 1402, 1190, 1085, 1030, 989, $963 \mathrm{~cm}^{-1} .{ }^{1} \mathrm{H}$ NMR $\left(500 \mathrm{~Hz}, \mathrm{CDCl}_{3}\right): \delta 6.36(\mathrm{dd}, J=10.4,17.6 \mathrm{~Hz}, \mathrm{H}), 6.25(\mathrm{dd}, J=1.0,17.7$ $\mathrm{Hz}, \mathrm{H}), 5.89$ (dd, $J=1.1,10.5 \mathrm{~Hz}, \mathrm{H}), 4.12$ (m, H), 2.94 (d, $J=3.5 \mathrm{~Hz}, \mathrm{H}), 2.77-2.67$ (m, 2H), 1.52-1.35 $(\mathrm{m}, 3 \mathrm{H}), 1.30-1.20(\mathrm{~m}, 2 \mathrm{H}), 0.93-0.87(\mathrm{~m}, 6 \mathrm{H}) .{ }^{13} \mathrm{C} \mathrm{NMR}\left(125 \mathrm{MHz}, \mathrm{CDCl}_{3}\right): \delta 201.9,136.7,129.1,68.5$, 46.0, 42.7, 21.8, 21.4, 11.7, 11.6. Chiral HPLC assay: $\mathrm{t}_{\mathrm{r}}=15.9 \mathrm{~min}$ (minor), 19.9 min (major) (Chiralcel $\mathrm{OD}, \lambda=230 \mathrm{~nm}$, heptane : isopropanol $=99 / 1,1.0 \mathrm{~mL} / \mathrm{min})$. HRMS calcd for $\mathrm{C}_{10} \mathrm{H}_{19} \mathrm{O}_{2}[\mathrm{M}+\mathrm{H}]$ : 171.1385 , found 171.1377 .<smiles>C=CC(=O)CC(O)C(C)O</smiles>

\section{Entry 7a, Table 1}

$[\alpha]_{\mathrm{D}}^{22}=+38.2\left(\mathrm{c} 1.02 \mathrm{CHCl}_{3}\right)$. IR (neat): 3480, 2956, 2990, 2895, 2858, 1682, 1616, 1472, 1403, 1257 , 1141, 1088, 996, 911, 836, $777 \mathrm{~cm}^{-1} .{ }^{1} \mathrm{H}$ NMR $\left(500 \mathrm{~Hz}, \mathrm{CDCl}_{3}\right): \delta 6.37(\mathrm{dd}, J=10.6,17.7 \mathrm{~Hz}, \mathrm{H}), 6.25$ $(\mathrm{dd}, J=0.8,17.6 \mathrm{~Hz}, \mathrm{H}), 5.89$ (dd, $J=1.0,10.6 \mathrm{~Hz}, \mathrm{H}), 3.92$ (dddd, $J=3.5,4.0,5.1,8.4, \mathrm{H}), 3.81$ (dq, $J$ $=5.1(\mathrm{~d}), 6.1(\mathrm{q}) \mathrm{Hz}, \mathrm{H}), 2.93(\mathrm{~d}, J=4.0 \mathrm{~Hz}, \mathrm{H}), 2.83(\mathrm{dd}, J=3.5,17.1 \mathrm{~Hz}, \mathrm{H}), 2.74(\mathrm{dd}, J=8.4,17.1 \mathrm{~Hz}$, $\mathrm{H}), 1.16(\mathrm{~d}, J=6.2 \mathrm{~Hz}, 3 \mathrm{H}), 0.89(\mathrm{~s}, 9 \mathrm{H}), 0.08(\mathrm{~s}, 3 \mathrm{H}), 0.07(\mathrm{~s}, 3 \mathrm{H}),{ }^{13} \mathrm{C} \mathrm{NMR}\left(125 \mathrm{MHz}, \mathrm{CDCl}_{3}\right): \delta$ $200.9,136.9,129.0,72.1,70.8,41.3,25.8,19.0,18.0,-4.4,-4.8$. HRMS calcd for $\mathrm{C}_{13} \mathrm{H}_{27} \mathrm{O}_{3} \mathrm{Si}[\mathrm{M}+\mathrm{H}]$, 258.1651, found 259.0 (low resolution).<smiles>C=CC(=O)CC(O)C(C)O</smiles>

\section{Entry $7 b$, Table 1}

$[\alpha]_{\mathrm{D}}{ }^{26}=-10.5\left(\mathrm{c} 1.32 \mathrm{CHCl}_{3}\right)$. IR (neat): 3481, 2956, 2930, 2896, 2858, 1683, 1616, 1473, 1403, 1256, 11141, 1094, 1005, 996, 836, $771 \mathrm{~cm}^{-1} .{ }^{1} \mathrm{H}$ NMR $\left(500 \mathrm{~Hz}, \mathrm{CDCl}_{3}\right): \delta 6.38(\mathrm{dd}, J=10.5,17.6 \mathrm{~Hz}, \mathrm{H}), 6.23$ $(\mathrm{dd}, J=1.1,17.6 \mathrm{~Hz}, \mathrm{H}), 5.88$ (dd, $J=1.0,10.6 \mathrm{~Hz}, \mathrm{H}), 3.94(\mathrm{~m}, \mathrm{H}), 3.82$ (dq, $J=4.2(\mathrm{~d}), 6.2(\mathrm{q}) \mathrm{Hz}, \mathrm{H})$, 2.78-2.71 (m, 2H), $1.17(\mathrm{~d}, J=6.2 \mathrm{~Hz}, 3 \mathrm{H}), 0.89(\mathrm{~s}, 9 \mathrm{H}), 0.08(\mathrm{~s}, 3 \mathrm{H}), 0.05(\mathrm{~s}, 3 \mathrm{H}) .{ }^{13} \mathrm{C} \mathrm{NMR}(125 \mathrm{MHz}$, $\left.\mathrm{CDCl}_{3}\right): \delta 200.3,136.8,128.9,71.5,70.3,42.1,25.8,19.2,18.0,-4.3$, -4.9. Anal Calcd for $\mathrm{C}_{13} \mathrm{H}_{26} \mathrm{O}_{3} \mathrm{Si}$, C:60.42, H:10.14, found C: 60.60, H: 10.12 .<smiles>C=CC(=O)CC(O)C(C)(C)Br</smiles>

Entry 8, Table 1

$[\alpha]_{\mathrm{D}}{ }^{26}=+33.7\left(\mathrm{c} 1.62 \mathrm{CHCl}_{3}\right) \cdot \mathrm{t}_{\mathrm{r}}=15.7$ (major), 19.7 (minor) min (Chiralcel AD, $\lambda=230 \mathrm{~nm}$, heptane : isopropanol = 98/2, $1 \mathrm{~mL} / \mathrm{min}$ ). IR (neat): 3490, 3030, 2975, 2360, 1679, 1615, 1497, 1453, 1401, 1147, 1088, $988 \mathrm{~cm}^{-1} .{ }^{1} \mathrm{H}$ NMR $\left(500 \mathrm{~Hz}, \mathrm{CDCl}_{3}\right): \delta$ 7.36-7.22 (m, 5H), $6.39(\mathrm{dd}, J=10.6,17.7 \mathrm{~Hz}, \mathrm{H}), 6.25(\mathrm{dd}$, $J=1.0,17.7 \mathrm{~Hz}, \mathrm{H}), 5.87(\mathrm{dd}, J=1.0,10.6 \mathrm{~Hz}, \mathrm{H}), 4.50\left(\mathrm{ABq}, v_{\mathrm{A}}=11.4, v_{\mathrm{B}}=22.0 \mathrm{~Hz}, 2 \mathrm{H}\right), 4.10(\mathrm{ddd}, J$ $=2.3,3.8,9.6 \mathrm{~Hz}, \mathrm{H}), 3.00(\mathrm{~d}, J=3.8 \mathrm{~Hz}, \mathrm{H}), 2.91(\mathrm{dd}, J=2.3,16.9 \mathrm{~Hz}, \mathrm{H}), 2.76(\mathrm{dd}, J=9.6,16.8 \mathrm{~Hz}$, $\mathrm{H}), 1.31(\mathrm{~s}, 3 \mathrm{H}), 1.28(\mathrm{~s}, 3 \mathrm{H}) .{ }^{13} \mathrm{C} \mathrm{NMR}\left(125 \mathrm{MHz}, \mathrm{CDCl}_{3}\right): \delta 201.0,139.2,136.8,129.0,77.1,73.3$, 63.9, 41.0, 21.8, 20.6. Anal Calcd for $\mathrm{C}_{15} \mathrm{H}_{20} \mathrm{O}_{3}, \mathrm{C}: 72.55$, H: 8.12, found C: 72.48, H: 8.12.

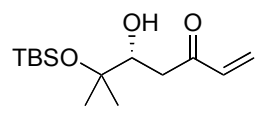

Entry 9, Table 1

$[\alpha]_{\mathrm{D}}{ }^{25}=+37.5\left(\mathrm{c} 1.34 \mathrm{CHCl}_{3}\right) . \mathrm{t}_{\mathrm{r}}=7.23$ (minor), 9.74 (major) min (Chiralcel OD, $\lambda=230 \mathrm{~nm}$, heptane : isopropanol = 99/1, $1 \mathrm{~mL} / \mathrm{min}$ ). IR (neat): 3508, 2956, 2991, 2888, 2858, 1682, 1617, 1472, 1403, 1363, 1256, 1180, 1154, 1081, 1044, 835, 813, $774 \mathrm{~cm}^{-1} .{ }^{1} \mathrm{H}$ NMR $\left(400 \mathrm{~Hz}, \mathrm{CDCl}_{3}\right): \delta 6.40(\mathrm{dd}, J=10.5,17.7$ $\mathrm{Hz}, \mathrm{H}), 6.24(\mathrm{dd}, J=1.0,17.7 \mathrm{~Hz}, \mathrm{H}), 5.88(\mathrm{dd}, J=1.0,10.5 \mathrm{~Hz}, \mathrm{H}), 3.84(\mathrm{ddd}, J=2.3,4.3,9.6 \mathrm{H}), 2.85$ $(\mathrm{dd}, J=2.4,16.5 \mathrm{~Hz}, \mathrm{H}), 2.82(\mathrm{~d}, J=4.1 \mathrm{~Hz}, \mathrm{H}), 2.66(\mathrm{dd}, J=9.8,16.5 \mathrm{~Hz}, \mathrm{H}), 1.25(\mathrm{~s}, 3 \mathrm{H}), 1.23(\mathrm{~s}, 3 \mathrm{H})$, 0.86 (s, 9H), 0.12 (s, 3H), $0.12(\mathrm{~s}, 3 \mathrm{H}),{ }^{13} \mathrm{C} \mathrm{NMR}\left(100 \mathrm{MHz}, \mathrm{CDCl}_{3}\right): \delta 201.1,136.9,128.9,75.4,75.0$, 40.9, 26.1, 25.8, 25.0, 18.0, -2.2. Anal. Calc. for $\mathrm{C}_{14} \mathrm{H}_{28} \mathrm{O}_{3} \mathrm{Si} \mathrm{C}: 61.72, \mathrm{H}: 10.36$, found C: 61.62, $\mathrm{H}$ : 10.16 . 


\section{Entry 10, Table 1}

$[\alpha]_{\mathrm{D}}^{26}=+31.0\left(\mathrm{c} 2.25 \mathrm{CHCl}_{3}\right)$. IR (neat): 3480, 2954, 1682, 1615, 1470, 1403, 1364, 1249, 1203, 1073, 990, 966, $879 \mathrm{~cm}^{-1} .{ }^{1} \mathrm{H}$ NMR $\left(500 \mathrm{~Hz}, \mathrm{CDCl}_{3}\right): \delta 6.33(\mathrm{dd}, J=10.4,17.7 \mathrm{~Hz}, \mathrm{H}), 6.23(\mathrm{dd}, J=1.1,17.7$ $\mathrm{Hz}, \mathrm{H}), 5.88(\mathrm{dd}, J=1.1,10.5 \mathrm{~Hz}, \mathrm{H}), 4.26(\mathrm{~m}, \mathrm{H}), 2.96(\mathrm{~d}, J=2.1 \mathrm{~Hz}, \mathrm{H}), 2.72(\mathrm{dd}, J=25.4,17.0 \mathrm{~Hz}$, H), $2.71(\mathrm{dd}, J=17.0,21.0 \mathrm{~Hz}, \mathrm{H}), 1.50(\mathrm{dd}, J=8.7,14.4 \mathrm{~Hz}, \mathrm{H}), 1.23(\mathrm{dd}, J=2.6,14.4 \mathrm{~Hz}, \mathrm{H}), 0.96$ (s, 9H). ${ }^{13} \mathrm{C} \mathrm{NMR}\left(125 \mathrm{MHz}, \mathrm{CDCl}_{3}\right): \delta 201.5,136.7,129.1,65.3,49.9,47.5,30.3,30.0$. Chiral HPLC assay: $\mathrm{t}_{\mathrm{r}}=17.5$ (minor), 19.1 (major) $\min$ (Chiralcel OD, $\lambda=230 \mathrm{~nm}$, heptane : isopropanol $=99.5 / 0.5$, $0.9 \mathrm{~mL} / \mathrm{min}$ ). HRMS calcd for $\mathrm{C}_{10} \mathrm{H}_{18} \mathrm{O}_{2}: 170.1307$, found 170.1299 , calcd for $\mathrm{C}_{6} \mathrm{H}_{13} \mathrm{O}$, [M- $\left.\mathrm{C}_{4} \mathrm{H}_{5} \mathrm{O}\right]$ : 101.0966, found 101.0957.

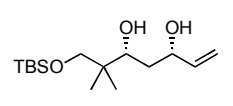

3a

To a solution of 7-t-butyldimethylsiloxy-5-hydroxy-6,6-dimethyl-hept-1-en-3-one (100 mg, $0.349 \mathrm{mmol})$ in dry tetrahydropyran $(3.2 \mathrm{~mL})$ at $-78^{\circ} \mathrm{C}$ was added anhydrous methanol $(0.8 \mathrm{~mL})$ and diethylmethoxyborane $\left(\mathrm{Et}_{2} \mathrm{~B}(\mathrm{OMe}), 0.384 \mathrm{~mL}\right.$ of $\left.0.1 \mathrm{M} \mathrm{THF}, 0.384 \mathrm{mmol}\right)$. The resulting mixture was stirred for $15 \mathrm{~min}$. Sodium borohydride $(14.5 \mathrm{mg}, 0.384 \mathrm{mmol})$ was then added portionwise under nitrogen and the mixture was stirred at $-78{ }^{\circ} \mathrm{C}$ for $3 \mathrm{~h}$. The reaction mixture was quenched with solid $\mathrm{Na}, \mathrm{K}$-tartrate (116 mg, 2 equiv) and was diluted with EtOAc and sat. solution of $\mathrm{NaHCO}_{3}$. the mixture was stirred overnight to ensure complete hydrolysis of intermediate boronate $\left(\mathrm{R}_{\mathrm{f}}=0.8\right.$, EtOAc:pet $\left.=1 / 4\right)$. The aqueous layer was extracted with EtOAc $(2 \mathrm{~mL} \times 3)$ and the combined organic layers were dried $\left(\mathrm{MgSO}_{4}\right)$, evaporated, and the residual oil was purified on a silica gel column $\left(\mathrm{Et}_{2} \mathrm{O}\right.$ :pet $\left.=1: 1\right)$ to afford $82.4 \mathrm{mg}(82 \%)$ as a single diastereomer.

$[\alpha]_{\mathrm{D}}{ }^{24}=+13.8\left(\mathrm{c} 1.80 \mathrm{CHCl}_{3}\right)$. IR (neat): 3406, 2957, 2803, 1472, 1362, 1256, 1093, 1007, 922, 837, $776,669 \mathrm{~cm}^{-1} .{ }^{1} \mathrm{H}$ NMR $\left(500 \mathrm{~Hz}, \mathrm{CDCl}_{3}\right): \delta 5.87(\mathrm{ddd}, J=5.9,10.5,17.1 \mathrm{~Hz}, \mathrm{H}), 5.28\left(\mathrm{td}, J=1.7^{\mathrm{t}}, 17.1^{\mathrm{d}}\right.$ $\mathrm{Hz}, \mathrm{H}), 5.07\left(\mathrm{td}, J=1.5^{\mathrm{t}}, 10.3^{\mathrm{d}} \mathrm{Hz}, \mathrm{H}\right), 4.36(\mathrm{~m}, \mathrm{H}), 4.32(\mathrm{~s} \mathrm{br}, 2 \mathrm{H}), 3.78(\mathrm{dd}, J=2.2,10.7 \mathrm{~Hz}, \mathrm{H}), 3.50$ $\left(\mathrm{ABq}, v_{\mathrm{A}}=9.8 \mathrm{~Hz}, v_{\mathrm{B}}=24.7 \mathrm{~Hz} 2 \mathrm{H}\right), 1.62\left(\mathrm{td}, J=2.6^{\mathrm{t}}, 14.2^{\mathrm{d}}, \mathrm{H}\right), 1.54(\mathrm{ddd}, J=9.3,10.5,14.0 \mathrm{~Hz}, \mathrm{H})$, $0.90(\mathrm{~s}, 9 \mathrm{H}), 0.88(\mathrm{~s}, 3 \mathrm{H}), 0.84(\mathrm{~s}, 3 \mathrm{H}), 0.08(\mathrm{~s}, 6 \mathrm{H}) .{ }^{13} \mathrm{C} \mathrm{NMR}\left(125 \mathrm{MHz}, \mathrm{CDCl}_{3}\right): \delta 140.9,114.0,80.6$, $73.5,73.4,38.0,37.9,25.7,22.2,19.0,18.0,-5.7$. LRMS calcd for $[\mathrm{M}+\mathrm{H}] \mathrm{C}_{15} \mathrm{H}_{33} \mathrm{O}_{3} \mathrm{Si}, 289.2$, found 289.4 .

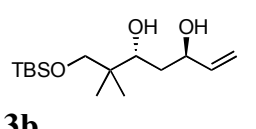

3b

To a flask containing tetramethylammonium triacetoxyborohydride $\left(\mathrm{Me}_{4} \mathrm{NBH}(\mathrm{OAc})_{3}\right.$ (490 $\mathrm{mg}, 1.86$ mmol) was added acetonitrile $(2 \mathrm{~mL})$ and $\mathrm{AcOH}(1 \mathrm{~mL})$ and the mixture was stirred at ambient temperature for $30 \mathrm{~min}$. After cooling the mixture to $-25{ }^{\circ} \mathrm{C}$, the 7-(tert-butyldimethylsilyloxy)-5hydroxy-6,6-dimethyl-hept-1-en-3-one $(66.6 \mathrm{mg}, 0.232 \mathrm{mmol})$ in acetonitrile $(1.0 \mathrm{~mL})$ was added and the mixture was stirred for $12 \mathrm{~h}$ at $-25{ }^{\circ} \mathrm{C}$. The mixture was quenched with a saturated solution of $\mathrm{Na}, \mathrm{K}-$ tartrate and was diluted with dichloromethane $(2 \mathrm{~mL})$, then the mixture was neutralized to $\mathrm{pH} 7 \mathrm{using} 2 \mathrm{~N}$ $\mathrm{NaOH}$ solution. After stirring $3 \mathrm{~h}$, the phase was separated and the aqueous phase was extracted with dichloromethane $(3 \mathrm{~mL} \times 5)$. The combined organic layers were dried $\left(\mathrm{MgSO}_{4}\right)$ and evaporated and the resulting oil was purified on silica gel chromatography $\left(\mathrm{Et}_{2} \mathrm{O}\right.$ :pet $\left.=1: 2\right)$ to afford $55.5 \mathrm{mg}(83 \%)$ of colorless oil. The anti/syn ratio was determined to be 90/10, as judged from ${ }^{1} \mathrm{H}$ NMR. $[\alpha]_{\mathrm{D}}{ }^{24}=+0.43$ (c $1.10 \mathrm{CHCl}_{3}$ ). IR (neat): 3411, 2952, 2811, 1470, 1362, 1054, 1005, $937 \mathrm{~cm}^{-1} .{ }^{1} \mathrm{H}$ NMR (500 Hz, $\left.\mathrm{CDCl}_{3}\right)$ : $\delta 5.94(\mathrm{ddd}, J=5.1,10.5,17.2 \mathrm{~Hz}, \mathrm{H}), 5.32\left(\mathrm{td}, J=1.7^{\mathrm{t}}, 17.2^{\mathrm{d}} \mathrm{Hz}, \mathrm{H}\right), 5.13\left(\mathrm{td}, J=1.7^{\mathrm{t}}, 10.5^{\mathrm{d}} \mathrm{Hz}, \mathrm{H}\right)$, $4.47(\mathrm{~m}, \mathrm{H}), 4.13(\mathrm{dd}, J=1.2,3.5 \mathrm{~Hz}, \mathrm{H}), 3.83(\mathrm{ddd}, J=2.1,3.6,11.0 \mathrm{~Hz}, \mathrm{H}), 3.50\left(\mathrm{ABq}, v_{\mathrm{A}}=9.7 \mathrm{~Hz}, v_{\mathrm{B}}\right.$ $=13.8 \mathrm{~Hz}, 2 \mathrm{H}), 3.18(\mathrm{~d}, J=6.5 \mathrm{~Hz}, \mathrm{H}), 1.73(\mathrm{ddd}, J=3.4,11.1,14.1 \mathrm{~Hz}, \mathrm{H}), 1.57(\mathrm{dd} b r, J=6.8,14.0$ $\mathrm{Hz}, \mathrm{H}), 0.89$ (s, 9H), $0.87(\mathrm{~s}, 3 \mathrm{H}), 0.81(\mathrm{~s}, 3 \mathrm{H}), 0.07(\mathrm{~s}, 6 \mathrm{H}) .{ }^{13} \mathrm{C} \mathrm{NMR}\left(125 \mathrm{MHz}, \mathrm{CDCl}_{3}\right): \delta 141.1$, $113.9,76.3,73.6,70.4,37.8,37.1,25.8,22.2,18.9,18.0,-5.71,-5.74$. LRMS calcd for $[\mathrm{M}+\mathrm{H}]$ $\mathrm{C}_{15} \mathrm{H}_{33} \mathrm{O}_{3} \mathrm{Si}$, 289.2, found 289.4. 


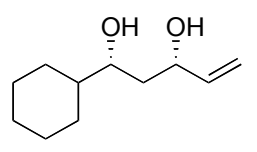

3c

A similar procedure was followed as described in the preparation of 3a (72\% yield as a single diastereomer). $[\alpha]_{\mathrm{D}}{ }^{24}=+7.85$ (c $2.88 \mathrm{CHCl}_{3}$ ). IR (neat): 3356, 2925, 2853, 1450, 1317, 1064, 990, 922, $893,850 \mathrm{~cm}^{-1} .{ }^{1} \mathrm{H}$ NMR $\left(400 \mathrm{~Hz}, \mathrm{CDCl}_{3}\right): \delta 5.84(\mathrm{ddd}, J=6.0,10.4,17.2 \mathrm{~Hz}, \mathrm{H}), 5.22\left(\mathrm{td}, J=1.5^{\mathrm{t}}, 17.2^{\mathrm{d}}\right.$ $\mathrm{Hz}, \mathrm{H}), 5.05\left(\mathrm{td}, J=1.4^{\mathrm{t}}, 10.4^{\mathrm{d}} \mathrm{Hz}, \mathrm{H}\right), 4.29(\mathrm{~m}, \mathrm{H}), 3.62(\mathrm{dd} \mathrm{br}, J=5.0,8.5 \mathrm{~Hz}, \mathrm{H}), 3.40(\mathrm{~s}, \mathrm{H}), 3.04(\mathrm{~s}$, H), 1.79-1.68 (m, 3H), 1.68-1.58 (m, 3H), 1.58-1.47 (m, H), 1.35-1.22 (m, H), 1.22-1.09 (m, 3H), 1.09$0.88(\mathrm{~m}, 2 \mathrm{H}) .{ }^{13} \mathrm{C}$ NMR $\left(100 \mathrm{MHz}, \mathrm{CDCl}_{3}\right): \delta 140.7,114.3,76.8,74.0,44.2,39.6,28.6,27.9,26.4,26.2$, 26.2. LRMS calcd for $[\mathrm{M}+\mathrm{H}] 185.1542$, found 185.2.

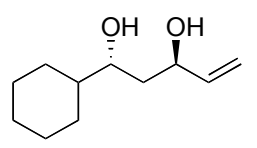

3d

A similar procedure was followed as described in the preparation of $\mathbf{3 a}$ ( $81 \%$ yield of a mixture of diastereomers, anti/syn $=91 / 9) .[\alpha]_{\mathrm{D}}{ }^{24}=-0.39\left(\mathrm{c} 1.28 \mathrm{CHCl}_{3}\right)$. IR (neat): 3363, 2926, 2853, 1450, 1416, 1062, 1039, 991, 921, $832 \mathrm{~cm}^{-1} .{ }^{1} \mathrm{H}$ NMR $\left(500 \mathrm{~Hz}, \mathrm{CDCl}_{3}\right): \delta 5.89$ (ddd, $\left.J=5.2,10.5,17.2 \mathrm{~Hz}, \mathrm{H}\right), 5.26$ $\left(\mathrm{td}, J=1.6^{\mathrm{t}}, 17.2^{\mathrm{d}} \mathrm{Hz}, \mathrm{H}\right), 5.10\left(\mathrm{td}, J=1.5^{\mathrm{t}}, 10.5^{\mathrm{d}} \mathrm{Hz}, \mathrm{H}\right), 4.43(\mathrm{~m}, \mathrm{H}), 3.64(\mathrm{ddd}, J=2.4,6.2,8.9 \mathrm{~Hz}, \mathrm{H})$, 3.30 (s br, H), 2.83 (s br, H), 1.86-1.80 (m, H), 1.78-1.68 (m, 3H), 1.68-1.58 (m, 3H), 1.36-1.28 (m, H), 1.28-1.04 (m, 3H), 1.04-0.80 (m, 2H). $\left.{ }^{13} \mathrm{C} \mathrm{NMR} \mathrm{(125} \mathrm{MHz,} \mathrm{CDCl}_{3}\right): \delta 140.7,114.1,73.0,70.4,43.6$, $39.1,28.8,28.2,26.4,26.1,26.0$.<smiles>CCCOCC(C)(C)CC(O)C1CC(c2ccccc2)=NO1</smiles>

\section{Entry 1, Table 2}

To a solution of starting diol $\mathbf{3 a}(24.1 \mathrm{mg}, 0.0835 \mathrm{mmol}$, dried azeotropically over benzene) in dichloromethane $(0.5 \mathrm{~mL})$ at $-78{ }^{\circ} \mathrm{C}$ was added ethylmagnesium bromide $(250 \mu \mathrm{L}$ of $1.0 \mathrm{M}$ soln in THF, $0.250 \mathrm{mmol}$ ) and stirred at this temperature for $20 \mathrm{~min}$. In a separate flask, a solution of benzohydroximoyl chloride $(19.4 \mathrm{mg}, 0.125 \mathrm{mmol})$ and isopropanol $(19.2 \mu \mathrm{L}, 0.25 \mathrm{mmol})$ in dichloromethane $(0.5 \mathrm{~mL})$ at $-78{ }^{\circ} \mathrm{C}$ was treated dropwise with ethylmagnesium bromide $(84 \mu \mathrm{L}$ of $1.0 \mathrm{M}$ soln in THF, $0.084 \mathrm{mmol})$. The solution of magnesium alkoxide prepared above was cannulated into the solution of nitrile oxide at $-78{ }^{\circ} \mathrm{C}$, stirred $30 \mathrm{~min}$, and kept at $-25^{\circ} \mathrm{C}$ for $10 \mathrm{~h}$. The reaction was quenched with half saturated $\mathrm{NH}_{4} \mathrm{Cl}$ (aq. soln) and extracted with $\mathrm{CH}_{2} \mathrm{Cl}_{2}(2 \mathrm{~mL}$ x 4). The combined organic layers were dried $\left(\mathrm{Na}_{2} \mathrm{SO}_{4}\right)$, filtered, and concentrated in vacuo. The residue was separated on a silica gel chromatography $($ EtOAc/Pet $=1 / 1)$ to give $20.0 \mathrm{mg}(59 \%)$ of 2-isoxalozine product as colorless oil. $[\alpha]_{\mathrm{D}}{ }^{24}=+98.9\left(\mathrm{c} 1.80 \mathrm{CHCl}_{3}\right)$. IR (neat): 3443, 2956, 2929, 2857, 1472, 1447, 1359, 1254, 1091, 910, $837 \mathrm{~cm}^{-1} .{ }^{1} \mathrm{H}$ NMR $\left(400 \mathrm{~Hz}, \mathrm{CDCl}_{3}\right): \delta 7.70-7.65(\mathrm{~m}, 2 \mathrm{H}), 7.43-7.38(\mathrm{~m}, 3 \mathrm{H}), 4.82(\mathrm{ddd}, J=3.8,8.1,10.1$ $\mathrm{Hz}, \mathrm{H}), 4.42(\mathrm{~d}, J=2.9 \mathrm{~Hz}, \mathrm{H}), 4.18(\mathrm{~d}, J=2.0 \mathrm{~Hz}, \mathrm{H}), 4.05(\mathrm{~m}, \mathrm{H}), 3.79(\mathrm{td}, J=3.0,10.1 \mathrm{~Hz}, \mathrm{H}), 3.49$ $\left(\mathrm{ABq}, v_{\mathrm{A}}=9.8, v_{\mathrm{B}}=15.4 \mathrm{~Hz}, 2 \mathrm{H}\right), 3.42(\mathrm{dd}, J=8.1,16.8 \mathrm{~Hz}, \mathrm{H}), 3.34(\mathrm{dd}, J=11.0,16.8 \mathrm{~Hz}, \mathrm{H}), 1.73-$ $1.61(\mathrm{~m}, 2 \mathrm{H}), 0.89(\mathrm{~s}, 9 \mathrm{H}), 0.88(\mathrm{~s}, 3 \mathrm{H}), 0.84(\mathrm{~s}, 3 \mathrm{H}), 0.07(\mathrm{~s}, 3 \mathrm{H}), 0.06(\mathrm{~s}, 3 \mathrm{H}) .{ }^{13} \mathrm{C} \mathrm{NMR}(100 \mathrm{MHz}$, $\left.\mathrm{CDCl}_{3}\right): \delta 156.8,130.0,129.5,128.6,126.7,82.8,80.3,73.5,72.7,38.1,36.0,32.4,25.7,22.2,18.9,18.0$, -5.7. Anal calcd for $\mathrm{C}_{22} \mathrm{H}_{37} \mathrm{NO}_{4} \mathrm{Si}, \mathrm{C}: 64.82, \mathrm{H}: 9.15, \mathrm{~N}: 3.44$, found C: $65.00, \mathrm{H}: 8.91, \mathrm{~N}: 3.29$.<smiles>CC(C)(COC(C)(C)C)CC(O)C1CC(c2ccccc2)=NO1</smiles>

\section{Entry 2, Table 2}

A procedure described for the preparation Entry 3, Table 2 was similarly followed to give $71 \%$ of product as colorless oil. $[\alpha]_{\mathrm{D}}{ }^{24}=-61.5\left(\mathrm{c} 2.1 \mathrm{CHCl}_{3}\right)$. IR (neat): 3447, 2956, 2929, 2857, 1472, 1447 , 1393, 1359, 1254, 1077, 1006, 938, 910, $836 \mathrm{~cm}^{-1}$. ${ }^{1} \mathrm{H}$ NMR $\left(400 \mathrm{~Hz}, \mathrm{CDCl}_{3}\right): \delta$ 7.70-7.62 (m, 2H), 7.42$7.35(\mathrm{~m}, 3 \mathrm{H}), 4.68$ (ddd, $J=4.3,8.2,12.7 \mathrm{~Hz}, \mathrm{H}), 4.04$ (s br, H), 3.98 (d, $J=7.6 \mathrm{~Hz}, \mathrm{H}), 3.87(\mathrm{~d}, J=10.7$ $\mathrm{Hz}, \mathrm{H}), 3.51\left(\mathrm{ABq}, v_{\mathrm{A}}=9.8, v_{\mathrm{B}}=16.5 \mathrm{~Hz}, 2 \mathrm{H}\right), 3.39(\mathrm{dd}, J=10.7,16.6 \mathrm{~Hz}, \mathrm{H}), 3.32(\mathrm{dd}, J=8.4,16.6$ $\mathrm{Hz}, \mathrm{H}), 2.31$ (s br, H), 1.73 (ddd, $J=1.6,10.1,13.9 \mathrm{~Hz}, \mathrm{H}), 1.55$ (ddd, $J=2.5,11.0,13.6 \mathrm{~Hz}, \mathrm{H}), 0.92$ (s, 
$3 \mathrm{H}), 0.90(\mathrm{~s}, 9 \mathrm{H}), 0.84$ (s, 3H), $0.079,0.078$ (s, 3H each). ${ }^{13} \mathrm{C} \mathrm{NMR}\left(100 \mathrm{MHz}, \mathrm{CDCl}_{3}\right): \delta 157.1,130.1$, 129.3, 128.6, 126.7, 84.4, 75.4, 73.9, 69.8, 37.8, 37.3, 35.9, 25.8, 22.3, 18.5, 18.1, -5.7, -5.8. Anal calcd for $\mathrm{C}_{22} \mathrm{H}_{37} \mathrm{NO}_{4} \mathrm{Si}, \mathrm{C}: 64.82, \mathrm{H}: 9.15, \mathrm{~N}: 3.44$, found $\mathrm{C}: 64.94, \mathrm{H}: 9.21, \mathrm{~N}: 3.49$.<smiles>CC(C)C1=NOC(CC(O)C2CCCCC2)C1</smiles>

Entry 3, Table 2

Representative procedure: A solution of oxime corresponding to $4 \mathrm{~b}(61.0 \mathrm{mg}, 0.300 \mathrm{mmol})$ was treated with ${ }^{\mathrm{t}} \mathrm{BuOCl}(32.6 \mathrm{mg}, 3.00 \mathrm{mmol})$ in dichloromethane $(1.0 \mathrm{~mL})$ for $30 \mathrm{~min}$ at $-78{ }^{\circ} \mathrm{C}$. In a separate flask, a mixture of cis-diol $3 \mathbf{c}(36.8 \mathrm{mg}, 0.200 \mathrm{mmol})$ and isopropanol $(45.9 \mu \mathrm{L}, 0.60 \mathrm{mmol})$ in dichloromethane $(1.0 \mathrm{~mL})$ was treated with ethylmagnesium bromide $(600 \mu \mathrm{L}, 1.0 \mathrm{M}$ in THF, $0.60 \mathrm{mmol})$ at $-78{ }^{\circ} \mathrm{C}$ and stirred for $10 \mathrm{~min}$. The solution of nitrile oxide solution formed above was transferred into this mixture dropwise at $\mathrm{rt}$. After $8 \mathrm{~h}$ at $\mathrm{rt}$, the mixture was treated with half saturated $\mathrm{NH}_{4} \mathrm{Cl}$ (aq. soln) and extracted with $\mathrm{CH}_{2} \mathrm{Cl}_{2}(2 \mathrm{~mL} \times 4)$. The combined organic layers were dried $\left(\mathrm{Na}_{2} \mathrm{SO}_{4}\right)$, filtered, and concentrated in vасио. The residue was separated on a silica gel chromatography $(\mathrm{EtOAc} / \mathrm{Pet}=1 / 1)$ to give $53.4 \mathrm{mg}$ (69\%) of dihydroisoxazole as colorless oil.

$[\alpha]_{\mathrm{D}}{ }^{24}=+19.6\left(\mathrm{c} 1.31 \mathrm{CHCl}_{3}\right)$. IR (neat): 3384, 2926, 1674, 1634, 1558, 1455, 1258, 1218, 1093, 953, 834, $778 \mathrm{~cm}^{-1} .{ }^{1} \mathrm{H}$ NMR $\left(400 \mathrm{~Hz}, \mathrm{CDCl}_{3}\right): \delta 4.71(\mathrm{q}, J=7.1 \mathrm{~Hz}, \mathrm{H}), 4.50(\mathrm{ddd}, J=5.5,7.1,10.8 \mathrm{~Hz}, \mathrm{H})$, $3.78(\mathrm{ddd}, J=3.2,5.5,9.3 \mathrm{~Hz}, \mathrm{H}), 3.63(\mathrm{ddd}, J=2.6,5.4,7.9 \mathrm{~Hz}, \mathrm{H}), 3.05$ (dd, $J=10.8,17.4 \mathrm{~Hz}, \mathrm{H})$, $2.89(\mathrm{dd}, J=7.3,17.5 \mathrm{~Hz}, \mathrm{H}), 1.82-1.70(\mathrm{~m}, 3 \mathrm{H}), 1.70-1.62(\mathrm{~m}, 3 \mathrm{H}), 1.62-1.52(\mathrm{~m}, \mathrm{H}), 1.34(\mathrm{~d}, J=6.7$ $\mathrm{Hz}, 3 \mathrm{H}), 1.28-1.08(\mathrm{~m}, 4 \mathrm{H}), 1.08-0.92(\mathrm{~m}, 3 \mathrm{H}), 0.87(\mathrm{~s}, 9 \mathrm{H}), 0.08(\mathrm{~s}, 3 \mathrm{H}), 0.06(\mathrm{~s}, 3 \mathrm{H}) .{ }^{13} \mathrm{C}$ NMR $(100$ $\left.\mathrm{MHz}_{,} \mathrm{CDCl}_{3}\right): \delta 162.4,82.5,76.5,73.7,64.8,44.1,35.1,34.6,28.7,27.8,26.4,26.2,26.1,25.6,22.1$, 18.0, -4.8, -5.0. Anal calcd for $\mathrm{C}_{20} \mathrm{H}_{39} \mathrm{NO}_{4} \mathrm{Si}, \mathrm{C}: 62.29, \mathrm{H}: 10.19, \mathrm{~N}: 3.63$, found C: $62.35, \mathrm{H}: 10.24, \mathrm{~N}$ : 3.65 .<smiles>CC(C)C1=NOC(CC(O)C2CCCCC2)C1</smiles>

\section{Entry 4, Table 2}

A similar procedure to the one for the preparation of Entry 1, Table 2 was followed. (60\% yield (87\% brsm, estimated from the inseparable mixture of starting material and the desired product, in a molar ratio of 33:67. The yield is based on the estimation from NMR spectra of mixture.) IR (neat): 3396, 2928, 2854, 1635, 1372, 1337, 1258, 1093, 1028, 952, 877, 834, $778 \mathrm{~cm}^{-1} .{ }^{1} \mathrm{H} \mathrm{NMR}\left(500 \mathrm{~Hz}, \mathrm{CDCl}_{3}\right): \delta 4.72(\mathrm{q}$, $J=6.5 \mathrm{~Hz}, \mathrm{H}), 4.47$ (ddd, $J=4.9,7.8,12.8 \mathrm{~Hz}, \mathrm{H}), 3.83(\mathrm{~m}, \mathrm{H}), 3.68$ (m, H), 3.05 (dd, $J=10.7,17.2 \mathrm{~Hz}$, H), $2.90(\mathrm{dd}, J=7.9,17.2 \mathrm{~Hz}, \mathrm{H}), 2.52(\mathrm{~d}, J=6.4 \mathrm{~Hz}, \mathrm{H}), 2.14(\mathrm{~d}, J=5.0 \mathrm{~Hz}, \mathrm{H}), 1.80-1.70(\mathrm{~m}, 3 \mathrm{H})$, $1.70-1.60(\mathrm{~m}, 3 \mathrm{H}), 1.56(\mathrm{ddd}, J=2.7,5.6,12.5 \mathrm{~Hz}, \mathrm{H}), 1.33$ (d, $J=6.4 \mathrm{~Hz}, 3 \mathrm{H}), 1.30-1.10(\mathrm{~m}, 4 \mathrm{H}), 1.10-$ $0.93(\mathrm{~m}, 3 \mathrm{H}), 0.88(\mathrm{~s}, 9 \mathrm{H}), 0.08(\mathrm{~s}, 3 \mathrm{H}), 0.06(\mathrm{~s}, 3 \mathrm{H}) .{ }^{13} \mathrm{C} \mathrm{NMR}\left(125 \mathrm{MHz}, \mathrm{CDCl}_{3}\right): \delta 163.0,83.3,72.8$, $70.6,44.0,37.1,35.5,29.2,28.5,26.7,26.4,26.3,25.9,22.6,18.2,-4.6,-4.7$. Anal calcd for $\mathrm{C}_{20} \mathrm{H}_{39} \mathrm{NO}_{4} \mathrm{Si}, \mathrm{C}: 62.29, \mathrm{H}: 10.19, \mathrm{~N}: 3.63$, found $\mathrm{C}: 62.25, \mathrm{H}: 10.16, \mathrm{~N}: 3.42$.

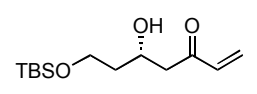

5

${ }^{1} \mathrm{H}$ NMR $\left(400 \mathrm{~Hz}, \mathrm{CDCl}_{3}\right): \delta 6.37(\mathrm{dd}, J=10.4,17.7 \mathrm{~Hz}, \mathrm{H}), 6.25(\mathrm{dd}, J=1.1,17.7 \mathrm{~Hz}, \mathrm{H}), 5.89$ (dd, $J=$ 1.1, $10.4 \mathrm{~Hz}, \mathrm{H}), 4.15(\mathrm{~m}, \mathrm{H}), 3.63(\mathrm{dd}, J=5.0,10.0 \mathrm{~Hz}, \mathrm{H}), 3.58(\mathrm{dd}, J=5.1,10.1 \mathrm{~Hz}, \mathrm{H}), 2.94(\mathrm{~d}, J=$ $4.4 \mathrm{~Hz}, \mathrm{H}), 2.79(\mathrm{~d}, J=6.1 \mathrm{~Hz}, 2 \mathrm{H}), 0.89(\mathrm{~s}, 9 \mathrm{H}), 0.07(\mathrm{~s}, 3 \mathrm{H}), 0.06(\mathrm{~s}, 3 \mathrm{H}),{ }^{13} \mathrm{C} \mathrm{NMR}(100 \mathrm{MHz}$, $\left.\mathrm{CDCl}_{3}\right): \delta 200.2,136.8,129.1,68.3,66.2,42.2,25.8,18.2,-5.41,-5.43$.

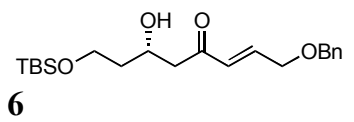

To a solution of Grubbs (II) catalyst $(4.0 \mathrm{mg}, 4.68 \mu \mathrm{mol})$ in $\mathrm{CH}_{2} \mathrm{Cl}_{2}(0.5 \mathrm{~mL})$ was added allyl benzylether $(41.7 \mathrm{mg}, 0.274 \mathrm{mmol})$, followed by portionwise addition of vinyl ketone 5 (22.9 $\mathrm{mg}, 0.0937 \mathrm{mmol})$ in $\mathrm{CH}_{2} \mathrm{Cl}_{2}(0.5 \mathrm{~mL})$. The resulting solution was stirred under reflux for $4 \mathrm{~h}$. The residue was directly loaded onto a silica gel column and eluted with EtOAc/pet $=1 / 4$ to give $15.9 \mathrm{mg}(65 \%)$ of 6 as pale brown oil. ${ }^{1} \mathrm{H}$ NMR $\left(500 \mathrm{~Hz}, \mathrm{CDCl}_{3}\right): \delta 7.40-7.35(\mathrm{~m}, 5 \mathrm{H}), 6.87\left(\mathrm{td}, J=4.3^{\mathrm{t}}, 16.0^{\mathrm{d}} \mathrm{Hz}, \mathrm{H}\right), 6.42\left(\mathrm{td}, J=2.1^{\mathrm{t}}, 16.0^{\mathrm{d}}\right.$ $\mathrm{Hz}, \mathrm{H}), 4.57$ (s, 2H), 4.21 (s, 2H), 4.14 (quintet, $J=5.8 \mathrm{~Hz}, \mathrm{H}), 3.63$ (dd, $J=5.1 \mathrm{~Hz}, \mathrm{H}), 3.57$ (dd, $J=5.9$, $10.0 \mathrm{~Hz}, \mathrm{H}), 2.77(\mathrm{~d}, J=5.9 \mathrm{~Hz}, 2 \mathrm{H}), 0.90(\mathrm{~s}, 9 \mathrm{H}), 0.070(\mathrm{~s}, 3 \mathrm{H}), 0.066(\mathrm{~s}, 3 \mathrm{H}) .{ }^{13} \mathrm{C} \mathrm{NMR}(125 \mathrm{MHz}$, 
$\left.\mathrm{CDCl}_{3}\right): \delta 199.8,143.4,137.8,129.8,128.8,128.2,128.0,73.2,69.0,68.6,66.5,43.5,26.1,18.5,-$ 5.1.HRMS calcd for $\mathrm{C}_{20} \mathrm{H}_{32} \mathrm{O}_{4} \mathrm{Si}[\mathrm{M}+\mathrm{H}], 365.2148$, found 365.2139. 